\title{
Zoonoses relacionadas ao trabalho: riscos biológicos associados ao manejo da vida silvestre no bioma Cerrado
}

\author{
Work-related zoonoses: biological risks associated with wildlife management in the Cerrado \\ biome
}

\author{
L. M. P. Carrijo, J. E. Limongi* \\ Programa de Pós-graduação em Saúde Ambiental e Saúde do Trabalhador, Universidade Federal de Uberlândia, \\ 38408-100, Uberlândia-MG, Brasil
}

*jeanlimongi@gmail.com

(Recebido em 19 de agosto de 2019; aceito em 02 de outubro de 2019)

\begin{abstract}
O contato frequente com animais aumenta o risco de infecções zoonóticas, sobretudo entre os profissionais relacionados ao manejo de vida silvestre. $\mathrm{O}$ objetivo deste trabalho foi analisar entre profissionais que lidam com fauna silvestre as principais atividades de riscos, o conhecimento sobre zoonoses, normas de biossegurança, ocorrência de treinamentos, além de descrever as principais doenças zoonóticas associadas aos animais por eles relatados. Foi realizado um estudo transversal entre servidores púbicos municipais, estaduais e federais do município de Uberlândia que manejam animais silvestres. Houve a participação de 173 profissionais. Apenas 56,1\% teve treinamento para manejo de animais. O conhecimento sobre doenças zoonóticas apresentou frequência de $47,9 \%$. O transporte $(54,3 \%)$ e a contenção/apreensão $(64,2 \%)$ são os principais tipos de contato. Os veículos e equipamentos de proteção individual são adequados em $34,2 \%$ e $47,5 \%$ dos casos, respectivamente. O uso de equipamento de proteção individual mostrou-se menos frequente entre os profissionais com maior tempo de trabalho nas diversas funções. Intervenções devem ser implementadas, sobretudo adotando medidas de promoção e prevenção de zoonoses e disseminação de conhecimento sobre estas patologias.

Palavras-chave: Zoonoses, Animais silvestres, Doenças relacionadas ao trabalho.
\end{abstract}

Frequent contact with animals increases the risk of zoonotic infections, especially among professionals involved in wildlife management. The objective of this study is to analyze among professionals dealing with wildlife the main activities of risk, knowledge about zoonoses, biosafety norms, and to describe the main zoonotic diseases associated with the animals reported by them. A cross - sectional study was carried out between municipal, state and federal public professionals from the municipality of Uberlândia who work with wildlife. A total of 173 professionals participated of the study. Only $56.1 \%$ had training to work with animals. Knowledge about zoonotic diseases presented a frequency of $47.9 \%$. Transport $(54.3 \%)$ and containment/seizure (64.2\%) are the main types of contact. Vehicles and personal protective equipment are adequate in $34.2 \%$ and $47.5 \%$ of cases, respectively. The use of personal protective equipment was less frequent among professionals with longer working time in the various functions.Interventions should be implemented, especially by adopting measures to promote and prevent zoonoses and disseminate knowledge about these pathologies.

Key words: Zoonoses, Animals, wild. Work-related diseases.

\section{INTRODUÇÃO}

Um animal afastado de seu habitat natural torna-se potencialmente um problema, seja no meio urbano, rural ou silvestre, principalmente pelo intercâmbio de doenças entre homens, outros animais silvestres e animais domésticos, as chamadas zoonoses [1]. Estas doenças são alvo de muita discussão no meio acadêmico e na mídia devido ao risco pertinente a elas de causarem epidemias ou se perpetuarem em um processo endêmico [2].

Conforme Karesh et al. (2012) [3], mais que 60\% das doenças infecciosas que acometem humanos são causadas por agentes etiológicos encontrados em várias espécies de animais silvestres e domésticos [3,4]. Além disso, mais de $70 \%$ desses eventos zoonóticos são causados por patógenos originários da vida silvestre [5].

Os animais silvestres que perdem seu habitat natural têm um destino indefinido, podem morrer, serem soltos de forma inadequada e em ambientes impróprios ou encaminhados para órgãos 
ambientais onde podem ser reabilitados e devidamente destinados [6]. A exposição frequente entre profissionais veterinários, biólogos, tratadores, policiais ambientais, bombeiros entre outros e os animais silvestres, aumenta o risco de infecções zoonóticas imprevisíveis e deixam esses profissionais mais susceptíveis que a população em geral [7]. Além destes profissionais, toda a população se encontra ameaçada, o que se agravou no último século com a globalização e com isso a maior velocidade e frequência com que os transportes aéreos, terrestres e aquáticos transfronteiriços ocorriam, o que permitiu além do trânsito de pessoas e produtos, também o de animais silvestres e patógenos emergentes [8]. Muitos destes patógenos, que anteriormente infectavam apenas animais, transpuseram a barreira de espécie e passaram a infectar seres humanos [9]. Soma-se a isso, as mudanças mundiais nas florestas, terras agrícolas e em cursos de água que afetam sobremaneira o espaço ecológico das doenças e consequentemente ocasiona o estreitamento do contato do homem com reservatórios animais, vetores e patógenos [10, 11].

Para profissionais que possuem estreito contato com animais silvestres, é imprescindível que os conceitos e ações de biossegurança para a garantia de um ambiente de trabalho seguro sejam implementados [12].

Em decorrência disso, existe a necessidade de se conhecer sobre os acidentes biológicos entre profissionais que manejam animais silvestres, como: incidência, animais envolvidos, complicações decorrentes destes acidentes, grau de conhecimento de riscos dos profissionais e medidas de prevenção.

Este estudo analisou entre profissionais que lidam com fauna silvestre no bioma cerrado, as principais atividades de riscos, o conhecimento sobre zoonoses, normas de biossegurança, além de descrever as principais doenças zoonóticas associadas aos animais por eles relatados.

\section{MATERIAL E MÉTODOS}

Foi realizado um estudo individuado observacional transversal. Para tanto, foram convidados a participarem todos os profissionais servidores públicos (municipais, estaduais e federais) que lidam diretamente com animais silvestres no município de Uberlândia-MG. Pelo fato deste contingente não ser amplo, propusemos o estudo em forma de censo. Ao todo, o presente estudo incluiu 255 profissionais alocados nas seguintes instituições: 17 profissionais no Instituto Brasileiro do Meio Ambiente e dos Recursos Naturais Renováveis (IBAMA); 15 Policiais Militares na Polícia Militar Ambiental; 11 profissionais na Universidade Federal de Uberlândia (Curso de Medicina Veterinária e Ciências Biológicas); 170 Bombeiros Militares no Corpo de Bombeiros Militar de Minas Gerais (CBMMG); 13 profissionais no Instituto Estadual de Florestas (IEF); 17 profissionais no Setor de Animais peçonhentos e quirópteros da Unidade de Vigilância de Zoonoses de Uberlândia (UVZ) e 12 profissionais no Zoológico Municipal de Uberlândia.

As informações acerca do total de profissionais, em cujas atribuições estavam incluso o contato com animais silvestres, foram fornecidas pela chefia responsável por cada instituição coparticipante. Foram excluídos dessa pesquisa os profissionais que trabalham nas referidas instituições que não mantinha contato direto com animais silvestres, bem como aqueles que se negaram a participar da pesquisa ou de assinar o Termo de Consentimento Livre e Esclarecido.

Os profissionais foram contatados por meio da chefia imediata e posteriormente, na presença do pesquisador principal, responderam às questões de um instrumento de coleta de dados semiestruturado, elaborado pelos pesquisadores com base em suas experiências no assunto. Foram pesquisadas as variáveis sociodemográficas sexo, idade, tempo de trabalho, profissão e instituição que trabalha, além das variáveis associadas às zoonoses e ao manejo de animais silvestres, quais sejam: "Conhece o conceito de animal silvestre?", "Conhece o conceito de animal exótico?", "Conhece o conceito de animal sinantrópico?, "Conhece o conceito de animal doméstico?", "Treinamento para manejo de animais silvestres?", "Conhece algum tipo de zoonose?", "Tem contato frequente com animais silvestres?", "Frequência de contato", "Situação de contato", "Estado dos animais durante a manipulação", "Tipo de contato", "Veículo apropriado no transporte de animal silvestre?", "Equipamentos apropriados na contenção de animal silvestre"?, "Uso de EPI no manejo de animais silvestres?", "Com quais Animais ou grupos de animais (Hospedeiros silvestres) possui maior contato na rotina de trabalho?" 
Os dados foram digitados em um banco de dados construído especificamente para este estudo no programa computacional Epi Info 7. 1. 3. Após a entrada dos dados, foi realizada a seguinte análise: (i) Distribuição da frequência das variáveis coletadas para a população pesquisada e verificação da consistência dos dados. Inicialmente, foi realizado a distribuição de frequências de todas as variáveis pesquisadas, caracterizando-se a população estudada segundo aspectos demográficos, socioeconômicos e fatores relacionados à exposição dos participantes. Posteriormente, verificou-se a consistência dos dados. (ii) Análise bivariada: Para as variáveis contínuas, foram calculadas as medidas de dispersão. Para associar atividades de risco de transmissão de zoonoses e o tempo de trabalho do profissional foram utilizados o teste não paramétrico teste de Kruskal-Wallis ou o teste t de Student, dependendo da normalidade dos dados $(\alpha=5 \%)$.

Essa pesquisa contou ainda com acesso aos Registros de Eventos de Defesa Social do $5^{\circ}$ Batalhão de Bombeiros Militar de Uberlândia no período de 2013 a 2017, nos quais são registradas as ocorrências atendidas por eles. Os dados foram filtrados por ocorrências relacionadas a acidentes entre os militares e animais.

Este estudo foi aprovado pelo Comitê de Ética em Pesquisa da Universidade Federal de Uberlândia, Minas Gerais, Brasil (Registro 1.301.743/2015).

\section{RESULTADOS}

Dos 255 profissionais lotados em instituições públicas no município de Uberlândia e que tinham entre as suas atribuições o contato com animais silvestres, um total de 173 (67,8\%) participaram da pesquisa, sendo a maior parte do sexo masculino (81,4\%), profissionais do Corpo de Bombeiros $(72,2 \%)$ e com tempo médio de trabalho de $11,3 \pm 8,1$ (Tabela 1 ).

Tabela 1: Características sócio demográficas dos participantes da pesquisa.

\begin{tabular}{lccc}
\hline Variáveis & n & \% & Média $( \pm$ DP) \\
\hline Sexo (n=172) & 140 & 81,4 & \\
Masculino & 32 & 18,6 & \\
Feminino & & & $36,3( \pm 9,1)$ \\
Idade (n=173) & & & $11,3( \pm 8,1)$ \\
Tempo de trabalho (n=173) & & & \\
Profissão (n=171) & 125 & 73,1 & \\
Bombeiro militar & 20 & 11,7 & \\
Assistente em saúde pública & 10 & 5,8 & \\
Policial militar ambiental & 8 & 4,7 \\
Médico veterinário & 8 & 4,7 \\
Outros (biólogo, engenheiro agrônomo, técnico em saúde & & & \\
ambiental) & 125 & 72,2 & \\
Instituição que trabalha (n=173) & 12 & 6,9 & \\
Corpo de Bombeiros Militar & 11 & 6,4 & \\
Unidade de Vigilância de Zoonoses & 11 & 6,4 & \\
Polícia Militar Ambiental & 8 & 4,6 & \\
Zoológico municipal & 6 & 3,5 & \\
Universidade Federal de Uberlândia & 0 & 0 & \\
IBAMA & &
\end{tabular}

Pouco mais de um terço dos profissionais conheciam o conceito de animal sinantrópico $(32,4 \%)$. Apesar dos profissionais terem tido contato frequente com animais silvestres $(92,4 \%)$, apenas $56,1 \%$ tiveram treinamento para manejo destes animais. O conhecimento sobre doenças zoonóticas apresentou uma frequência de $47,9 \%$ e apenas $4,2 \%$ afirmaram ter contraído uma zoonose (Tabela 2). 
Tabela 2: Conhecimentos sobre conceitos relacionados à fauna silvestre, doenças zoonóticas e caracterização do contato com animais silvestres.

\begin{tabular}{|c|c|c|}
\hline Variáveis & n & $\%$ \\
\hline Conhece o conceito de animal silvestre? $(n=173)$ & 143 & 82,7 \\
\hline Conhece o conceito de animal exótico? $(n=172)$ & 134 & 77,9 \\
\hline Conhece o conceito de animal sinantrópico? $(n=173)$ & 56 & 32,4 \\
\hline Conhece o conceito de animal doméstico? $(n=173)$ & 151 & 87,8 \\
\hline Treinamento para manejo de animais silvestres? $(n=166)$ & 93 & 56,1 \\
\hline Conhece algum tipo de zoonose? $(n=121)$ & 58 & 47,9 \\
\hline Já contraiu alguma zoonose? $(n=120)$ & 5 & 4,2 \\
\hline Tem contato frequente com animais silvestres? $(n=172)$ & 159 & 92,4 \\
\hline \multicolumn{3}{|l|}{ Frequência de contato $(n=148)$} \\
\hline Diariamente & 48 & 32,4 \\
\hline Semanalmente & 43 & 29,0 \\
\hline Mensalmente & 25 & 16,9 \\
\hline 1 a 3 episódios isolados anualmente & 18 & 12,2 \\
\hline 4 a 10 episódios isolados anualmente & 14 & 9,5 \\
\hline \multicolumn{3}{|l|}{ Situação de contato* $(n=173)$} \\
\hline Presos & 40 & 14,1 \\
\hline Soltos em seu habitat & 42 & 24,3 \\
\hline Soltos fora do habitat & 114 & 65,9 \\
\hline \multicolumn{3}{|l|}{ Estado dos animais durante a manipulação (em geral)* $(n=173)$} \\
\hline Saudáveis & 122 & 70,5 \\
\hline Doentes (presumível ou confirmada) & 82 & 47,4 \\
\hline Mortos & 3 & 1,7 \\
\hline \multicolumn{3}{|l|}{ Tipo de contato $*(n=173)$} \\
\hline Visual & 44 & 25,4 \\
\hline Transporte & 94 & 54,3 \\
\hline Contenção/apreensão & 111 & 64,2 \\
\hline Cuidados veterinários & 14 & 13,3 \\
\hline Manipulação para fins acadêmicos & 14 & 8,1 \\
\hline Veículo apropriado no transporte de animal silvestre? $(n=155)$ & 53 & 34,2 \\
\hline Equipamentos apropriados na contenção de animal silvestre? $(n=160)$ & 76 & 47,5 \\
\hline
\end{tabular}

Na maioria das vezes, os profissionais entravam em contato com os animais soltos e fora do seu habitat natural $(65,9 \%)$, com alta frequência de animais doentes $(47,4 \%)$. O transporte e a contenção/apreensão foram os principais tipos de contato, respondendo por 54,3\% e $64,2 \%$ dos casos, respectivamente. Apesar disso, os veículos e os equipamentos de proteção individual (EPI) foram adequados em apenas 34,2\% e 47,5\% dos casos, respectivamente (Tabela 2).

Em relação ao uso de EPI nas diversas atividades de risco de transmissão de zoonoses, foi avaliado a sua utilização comparando com o tempo médio de trabalho na função. Apesar de apenas a atividade Contenção/apreensão ter apresentando significância estatística $(\mathrm{p}=0,02)$, nota-se que para todas as atividades pesquisadas, àqueles que não utilizaram EPI sempre tinham média de tempo de trabalho maior (Tabela 3). 
Tabela 3: Relação entre o uso de EPI em atividades de risco de transmissão de doenças zoonóticas e o tempo de trabalho na função.

\begin{tabular}{|c|c|c|c|c|c|}
\hline \multicolumn{2}{|c|}{ Uso de EPI no manejo de animais silvestres } & \multirow{2}{*}{$\begin{array}{c}\mathbf{n} \\
114\end{array}$} & \multirow{2}{*}{$\begin{array}{c}\text { Tempo médio de } \\
\text { trabalho na } \\
\text { função (anos) }\end{array}$} & \multirow{2}{*}{$\begin{array}{c}\begin{array}{c}\text { Desvio } \\
\text { padrão }\end{array} \\
7,2\end{array}$} & \multirow{3}{*}{$\begin{array}{c}\text { Valor de } \\
p\end{array}$} \\
\hline Transporte $(n=139)$ & Sim & & & & \\
\hline & Não & 16 & 14,6 & 11,4 & \\
\hline \multirow[t]{2}{*}{ Contenção/apreensão (n=134) } & Sim & 119 & 10,8 & 7,4 & \multirow{2}{*}{$\mathbf{0 , 0 2} 2^{\mathrm{K}}$} \\
\hline & Não & 15 & 17,4 & 10,6 & \\
\hline \multirow[t]{2}{*}{ Alimentação (n=78) } & Sim & 43 & 12,2 & 8,1 & \multirow{2}{*}{0,91} \\
\hline & Não & 35 & 12,4 & 9,0 & \\
\hline \multirow[t]{2}{*}{ Higienização do animal $(n=56)$} & Sim & 14 & 11,7 & 10,3 & \multirow{2}{*}{0,80} \\
\hline & Não & 33 & 12,4 & 7,3 & \\
\hline \multirow[t]{2}{*}{ Higienização do cativeiro $(n=67)$} & Sim & 32 & 11,5 & 9,6 & \multirow{2}{*}{0,34} \\
\hline & Não & 35 & 13,6 & 8,1 & \\
\hline \multirow[t]{2}{*}{ Higienização do veículo (n=82) } & Sim & 50 & 11,1 & 8,0 & \multirow{2}{*}{0,09} \\
\hline & Não & 32 & 14,3 & 8,7 & \\
\hline \multirow[t]{2}{*}{ Cuidados a saúde do animal $(n=73)$} & Sim & 42 & 11,3 & 9,1 & \multirow{2}{*}{0,79} \\
\hline & Não & 31 & 11,8 & 6,7 & \\
\hline \multirow[t]{2}{*}{ Contato com secreções (n=102) } & Sim & 81 & 11,7 & 8,0 & \multirow{2}{*}{0,70} \\
\hline & Não & 21 & 12,5 & 8,8 & \\
\hline \multirow[t]{2}{*}{ Contato com excreções (n=93) } & Sim & 72 & 11,7 & 8,7 & \multirow{2}{*}{0,39} \\
\hline & Não & 21 & 13,6 & 8,7 & \\
\hline \multirow[t]{2}{*}{ Contato com sangue $(n=93)$} & Sim & 66 & 10,7 & 7,1 & \multirow{2}{*}{0,68} \\
\hline & Não & 27 & 11,4 & 7,8 & \\
\hline \multirow[t]{2}{*}{ Contato com fezes $(n=99)$} & Sim & 66 & 11,5 & 8,2 & \multirow{2}{*}{0,42} \\
\hline & Não & 33 & 13,0 & 8,5 & \\
\hline
\end{tabular}

${ }^{\mathrm{K}}$ Teste de Kruskal-Wallis. Demais análises teste t de Student. EPI: Equipamento de Proteção Individual.

A utilização de luvas foi a única medida de proteção consistentemente declarada pelos entrevistados, com frequência de $72 \%$. Vale ressaltar que o fardamento de uso corrente entre os militares, favorece proteção de vestuário e calçado e apesar de não terem sido citados por todos, a farda e o coturno foram incluídos nos resultados.

No tocante à ocorrência de acidentes decorrentes do contato dos profissionais inqueridos com animais silvestres no exercício de suas funções laborais, 140 acidentes foram descritos. Dentre esses constam picada de ectoparasitas (pulgas e carrapatos) $(53 ; 37,8 \%)$, arranhadura $(19 ; 13,6 \%)$, mordedura/bicada $(19 ; 13,6 \%)$, o contato direto com secreções/excreções $(16 ; 11,4 \%)$, fezes $(11$; $7,8 \%)$, sangue $(11 ; 7,8 \%)$ e acidentes com perfuro cortantes $(11 ; 7,8 \%)$.

Os dados obtidos com o acesso aos Registros de Eventos de Defesa Social do $5^{\circ}$ Batalhão de Bombeiros Militar de Uberlândia no período de 2013 a 2017 resumiram-se em apenas sete notificações, todas relacionadas a acidentes com animais peçonhentos. São elas: três picadas de escorpião, uma de marimbondo, uma de abelha e duas mordidas de cobra.

$\mathrm{Na}$ Tabela 4 estão apresentados os animais ou grupos de animais contatados pelos profissionais que participaram do estudo. As aves (16,3\%), serpentes $(14,6 \%)$ e tamanduás $(12,5 \%)$ foram os mais relatados. Estes animais, bem como algumas das respectivas doenças zoonóticas a eles relacionados são mostrados na Tabela 5 . 
Tabela 4: Frequência dos animais ou grupos de animais que os profissionais relataram maior contato na rotina de trabalho.

\begin{tabular}{lc}
\hline Animais ou grupos de animais (Hospedeiros silvestres) & N \\
\hline Aves & 121 \\
Serpentes & 108 \\
Tamanduás (Myrmecophaga tridactyla, Tamandua tetradactyla) & 92 \\
Ouriço-caixeiro (Coendou villosus) & 73 \\
Gambás (principalmente, Didelphis marsupialis) & 72 \\
Outros répteis (exceto serpentes) & 44 \\
Lobo Guará (Chrysocyon brachyurus) & 43 \\
Felinos silvestres & 38 \\
Capivara (Hydrochoerus hydrochaeris) & 38 \\
Canídeos silvestres (exceto Lobo Guará) & 31 \\
Primatas não humanos & 21 \\
Quati (Nasua nasua) & 17 \\
Morcegos & 9 \\
Tatus & 8 \\
Jaritataca (Conepatus semistriatus) & 8 \\
Roedores silvestres (exceto capivara) & 6 \\
Outros [Guaxinim (Procyon cancrivorus), Jupará (Potos flavus), Cervídeos] & 9 \\
\hline
\end{tabular}


Tabela 5: Algumas doenças que podem ser transmitidas aos humanos por contato direto com animais silvestres ou suas secreções/excreções.

Animais ou grupos de animais

Aves

Tamanduás

Gambás

Répteis

Felinos silvestres

Ouriços
Zoonoses (Bacterianas ${ }^{\mathrm{B}}$, Virais ${ }^{\mathrm{V}}$, Parasitárias ${ }^{\mathrm{P}}$, Fúngicas $^{\mathrm{F}}$, Ectoparasitárias ${ }^{\mathrm{E}}$ )

Criptosporidíase $^{\mathrm{P} ;}$ [13]

Doença de Newcastle $^{\mathrm{V} ;}$ [13]

Febre $\mathrm{Q}^{\mathrm{B} ;}[14]$

Giardiase $^{\mathrm{P} ;}[13]$

Influenza $\mathrm{A}^{\mathrm{V}}$ (gripe aviária) [14]

Listeriose $^{\mathrm{B} ;}[13]$

Psitacose/ clamidiose ${ }^{\mathrm{B} ;}$ [14]

Salmonelose $^{\mathrm{B} ;}$ [14]

Toxoplasmose $^{\mathrm{P} ;}[13]$

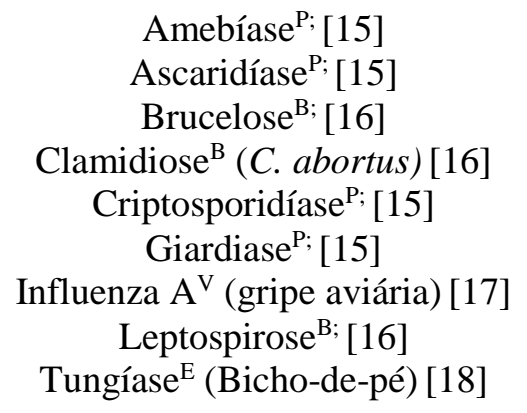

Doença de Lyme ${ }^{\mathrm{B} ;}$ [13]

Leptospirose $^{\mathrm{B} ;}$ [14]

Tricofitose $\mathrm{F}^{\mathrm{B}}[14]$

$$
\text { Anthrax }{ }^{\mathrm{B} ;}[14]
$$

Doença de chagas $^{\mathrm{P}}$ [14]

Infecções por mordida de gambá ${ }^{\mathrm{B} ;}$ [14]

Febre Maculosa $^{\mathrm{B} ;}$ [13]

Leptospirose $^{\mathrm{B} ;}$ [19]

Raiva ${ }^{\mathrm{V} ;}[14]$

Salmonelose ${ }^{\mathrm{B} ;}[14]$

Tuberculose ${ }^{\mathrm{B} ;}$ [14]

$$
\begin{gathered}
\text { Criptosporidíase }^{\mathrm{P} ;}[13] \\
\text { Giardiase }^{\mathrm{P} ;}[13] \\
\text { Leptospirose }^{\mathrm{B} ;}[19] \\
\text { Salmonelose }^{\mathrm{B} ;}[14]
\end{gathered}
$$

Anthrax $^{\mathrm{B} ;}[14]$

Clamidiose $^{\mathrm{B}}$ (C. felis) [14]

Criptosporidíase $^{\mathrm{P}}$ [14]

Dermatofitose por Microsporum ${ }^{4 ;}$ [14]

Dipilidiose $^{\mathrm{P} ;}[14]$

Escabiose $^{\mathrm{E}}$ (sarna) [13]

Giardiase $^{\mathrm{P} ;}[14]$

Larva Migrans Cutânea ${ }^{\mathrm{P}}$ (Bicho Geográfico) [14]

Leptospirose $^{\mathrm{B} ;}[20,21]$

Mordida de Carrapatos ${ }^{\mathrm{E} ;}$ [14]

Mordida de pulga ${ }^{\mathrm{E} ;}[14]$

Raiva ${ }^{\mathrm{V} ;}$ [13]

Salmonelose ${ }^{\mathrm{B} ;}$ [14]

Toxoplasmose $^{\mathrm{P} ;}[14]$ 
Capivara (Hydrochoerus hydrochaeris)

Canídeos silvestres

Primatas não humanos

\footnotetext{
Morcegos

Tatus

Roedores silvestres (exceto capivara)
}

Cervídeos

Guaxinim (Procyon cancrivorus)

Jaritataca (Conepatus semistriatus)

Quati (Nasua nasua)
Tricofitose $^{\mathrm{F} ;}[14]$

Tuberculose $^{\mathrm{B} ;}$ [14]

Escabiose $^{\mathrm{E}}$ (sarna) [22]

Febre Maculosa $^{\mathrm{B} ;}[13]$

Leptospirose $^{\mathrm{B} ;}[22]$

Raiva ${ }^{\mathrm{V} ;}[22]$

Anthrax ${ }^{\mathrm{B} ;}$ [14]

Criptosporidíase $^{\mathrm{P} ;}$ [14]

Dermatofitose por Microsporum ${ }^{\mathrm{E} ;}$ [14]

Dipilidiose $^{\mathrm{P} ;}[14]$

Doença de chagas ${ }^{\mathrm{P} ;}[14]$

Escabiose $^{\mathrm{E}}$ (sarna) [13]

Giardiase $^{\mathrm{P} ;}[13]$

Larva Migrans Cutânea ${ }^{\mathrm{P}}$ (Bicho Geográfico) [14]

Leptospirose $^{\mathrm{B} ;}[14]$

Mordida de Carrapatos ${ }^{\mathrm{E} ;}$ [14]

Mordida de pulga ${ }^{\mathrm{E} ;}[14]$

Raiva ${ }^{\mathrm{V} ;}[13]$

Salmonelose ${ }^{\mathrm{B} ;}[14]$

Tricofitose ${ }^{\mathrm{F} ;}[14]$

Tuberculose $^{\mathrm{B} ;}[14]$

Balantidiase $^{\mathrm{P} ;}[14]$

Bertielose $^{\mathrm{P} ;}[14]$

Criptosporidíase $^{\mathrm{P} ;}[14]$

Giardiase $^{\mathrm{P} ;}[13]$

Herpesviroses $^{\mathrm{V} ;}$ [13]

Oesophagostomoses $^{\mathrm{P} ;}[14]$

Sarampo $^{\mathrm{V} ;}[23]$

Tricofitose ${ }^{\mathrm{F} ;}[14]$

Tuberculose $^{\mathrm{B} ;}$ [14]

Raiva $^{\mathrm{V} ;}[13]$

Raiva ${ }^{\mathrm{V} ;}$ [13]

Hanseníase $^{\mathrm{B} ;}[23]$

Doença de Lyme ${ }^{\mathrm{B} ;}$ [13]

Encefalite Equina do Leste ${ }^{\mathrm{V} ;}$ [13]

Febre Maculosa $^{\mathrm{B} ;}$ [13]

Febre $\mathrm{Q}^{\mathrm{B} ;}[14]$

Giardiase $^{\mathrm{P} ;}[14]$

Hantavirose $^{\mathrm{V} ;}[14]$

Leptospirose $^{\mathrm{B} ;}[14]$

Listeriose $^{\mathrm{B} ;}[13]$

Peste $^{\mathrm{B} ;}[23]$

Raiva ${ }^{\mathrm{V} ;}$ [13]

Vaccinia $^{\mathrm{V} ;}[23]$

Anthrax $^{\mathrm{B} ;}$ [14]

Doença de Lyme ${ }^{\mathrm{B} ;}[13]$

Listeriose $^{\mathrm{B} ;}[13]$

Raiva ${ }^{\mathrm{V} ;}[13]$

Raiva ${ }^{\mathrm{V} ;}$ [13]

Tuberculose $^{\mathrm{B} ;}$ [14]

Raiva ${ }^{\mathrm{V} ;}$ [13] 


\section{DISCUSSÃO}

As complexas alterações ambientais, sejam elas provocadas por ação antrópica ou por desastres naturais, muitas vezes implicam no desequilíbrio de populações de animais silvestres. Os efeitos desses impactos, além da diminuição da biodiversidade, estendem-se à saúde pública e à economia, principalmente pela alteração na epidemiologia das doenças que possuem os animais silvestres como reservatórios, as zoonoses [13].

Uma das consequências do avanço das áreas urbanas para o meio rural é o aumento do número de animais silvestres em fuga ou em busca de comida e abrigo nas cidades. Os dados do Corpo de Bombeiros de Minas Gerais registraram um aumento gradativo desse tipo de ocorrência, de 2784 registros em 2005 para 7198 em 2015 [24]. Sendo assim, a preparação técnica (teórico/prática) dos profissionais, bem como a utilização de EPIs específicos para manejo de animais tornam-se ações primordiais no tocante à biossegurança.

A abordagem do presente estudo compreendeu avaliar a exposição de profissionais que têm como parte de suas atribuições, o contato com animais silvestres, constituindo um grupo de risco para aquisição de doenças zoonóticas. Dado a iminência com que este tipo de doença incide na população, a presente investigação justifica-se não apenas pela natureza severa da doença em si, mas também por abranger um grupo consideravelmente vulnerável por sua exposição. Neste estudo, apenas 58 profissionais $(47,9 \%)$ responderam ter conhecimento prévio de alguma doença zoonótica e 46 (38\%) citaram pelo menos uma zoonose conhecida. O desconhecimento destas doenças leva a uma percepção de risco subestimada e, por conseguinte, a diminuição de medidas preventivas [25].

Com relação a treinamento para manejo de animais silvestres, $56,1 \%$ dos profissionais que responderam a essa questão afirmaram já terem tido algum treinamento. Em notas extraordinárias de alguns questionários, foi relatado que os treinamentos são aleatórios e efêmeros, geralmente oferecidos por setores da Universidade Federal de Uberlândia. Uma iniciativa interessante veio do Corpo de Bombeiros de Minas Gerais que lançou uma Instrução Técnica Operacional (ITO) para a captura de animais, a qual tem por finalidade orientar as ações das guarnições dos Bombeiros Militares empenhadas neste tipo de ocorrência e padronizar o uso dos equipamentos de captura [24]. Em estudo semelhante nos Estados Unidos, que buscou descrever as exposições às doenças zoonóticas e identificar fatores e motivações a respeito do uso de medidas de proteção pessoal entre biólogos e veterinários que lidam com animais silvestres, apenas $38 \%$ dos profissionais relataram terem recebido algum treinamento a respeito de doenças zoonóticas [26].

A respeito do uso de EPI e medidas de segurança, enfatiza-se o uso de luvas na redução do risco de transmissão de patógenos por proteção de barreira [27], entretanto vários dos profissionais que relataram terem sido mordidos, afirmaram estarem usando luvas de couro e que os dentes dos animais as perfuraram. Considerando que $65,9 \%$ dos entrevistados afirmaram lidar principalmente com animais soltos e fora do habitat, o risco de acidentes é aumentado, devido às mudanças de comportamento do animal e necessidade de defesa [13].

As luvas estão disponíveis em uma variedade de materiais, como látex, nitrilo, vinil, couro, algodão, malha de aço e outros. A escolha das luvas depende da operação que se pretende realizar [28]. As luvas de couro ou de raspa são bastante resistentes e geralmente utilizadas para proteção contra agentes mecânicos (pesados, abrasivos, cortantes e perfurantes) [29]. Teoricamente, a escolha da luva pelos profissionais mordidos foi acertada, mas na prática ela não conferiu proteção adequada às mordidas de animais de dentes pontiagudos como, por exemplo, os do Gambá comum (Didelphis marsupialis). Segundo normas europeias, o teste para avaliação da resistência à perfuração consiste em medir a quantidade de força necessária para perfurar a luva com um ponto de tamanho padrão e a uma determinada velocidade $(10 \mathrm{~cm} / \mathrm{min})$. O nível de desempenho mais alto é 4, o que corresponde a uma força de $150 \mathrm{~N}$ [29]. Para conferir proteção contra mordidas deveria haver uma luva com desempenho 4 nos testes de resistência à perfuração e 5 nos testes de resistência a corte com lâminas [30]. Parece não haver nenhuma luva no mercado realmente eficaz na proteção de mordidas de animais, para as quais são atribuídos os riscos mecânico e biológico concomitantemente. A maioria das luvas designadas para proteção de agentes mecânicos possuem nível de desempenho 1 para corte com lâminas [31]. Por conseguinte, mostra-se imperioso que as técnicas para contenção de animais silvestres sejam constantemente revisadas, que os equipamentos 
utilizados sejam adequados e que os treinamentos sejam continuados e específicos para as diversas operações.

Não houve relatos a respeito do hábito de higienizar as mãos como medida de proteção utilizada entre os profissionais entrevistados. A higiene vigilante das mãos é a medida mais importante para reduzir o risco de transmissão de doenças zoonóticas. Os agentes patogênicos mais comuns são transmitidos pelo contato mão-boca diretamente de animais ou indiretamente por meio do ambiente contaminado [28].

Houve uma associação e tendências à associação entre a utilização de EPI e o tempo de trabalho nas várias funções em que se sucede o contato entre o profissional e animais silvestres. Pôde-se observar uma constância do não uso de EPI entre os profissionais que trabalham nas diversas funções há mais tempo. Embora a relação tenha sido estatisticamente significativa somente um uma das funções analisadas, supõem-se que o mesmo se repetiria nas demais funções, caso a amostra populacional tivesse sido maior. Em um estudo nos Estados Unidos se verificou uma associação inversa entre frequência de exposições de risco e uso de EPI. O tempo a que estão submetidos a exposições de risco ou a frequência com que elas ocorrem contribui para uma banalização da atividade, diminuição da percepção de risco e redução do uso de EPI [25]. A falta de educação permanente sobre o tema também pode ter afetado este comportamento entre os profissionais.

Vale ressaltar que o número reduzido de relatos de antecedentes de doenças zoonóticas entre os profissionais inqueridos, não deve incentivar a prática do trabalho sem segurança, já que muitas infecções zoonóticas são subclínicas, ou seja, não manifestam sintomas, e outras ainda podem tornar-se crônicas, podendo afetar a saúde do profissional no futuro [32].

Acidentes de trabalho relacionados com animais silvestres estão associados a uma maior probabilidade de infecção zoonótica [12]. Em uma revisão de literatura, foram constatados vários estudos soroepidemiológicos, casos clínicos e investigações de surtos de infecções e doenças zoonóticas entre veterinários [33]. Obviamente, outros profissionais que também lidam com esses animais silvestres, estão sujeitos em menor ou maior grau a essas infecções/doenças. Estes profissionais, de forma convergente, servem como sentinelas para agentes patogênicos emergentes e, divergentemente, podem espalhar patógenos zoonóticos para a comunidade e para os animais para os quais eles prestam cuidados [33, 34].

$\mathrm{O}$ acesso aos Registros de Eventos de Defesa Social (REDS) do corpo de Bombeiros Militares no período de 2013 a 2017, em que constavam apenas sete notificações de acidentes de trabalho entre militares sobre animais peçonhentos, revelou a subnotificação dos demais acidentes e suas potencialidades mórbidas. Em decorrência destes fatores acima mencionados, a relevância deste estudo se faz ainda mais proeminente, pois a acuidade laboral no lidar com animais silvestres parece ser insatisfatória, considerando uso de EPIs e treinamento periódico.

De fato, a carência de projetos de educação permanente e de treinamentos de forma sistemática e continuada, acerca do comportamento dos animais e das zoonoses por eles transmitidas afeta sobremaneira a biossegurança dos profissionais envolvidos no manejo de fauna silvestre. Agravante é que os servidores públicos não estão protegidos por leis federais específicas de segurança e medicina do trabalho, assim como os profissionais celetistas.

Portanto, os resultados aqui apresentados demonstraram que intervenções nos ambientes de trabalho devem ser implementadas, sobretudo promovendo medidas de promoção e prevenção de zoonoses e disseminação de conhecimento sobre estas patologias. Normatização específica pode auxiliar sobremaneira na padronização das condutas de biossegurança para os profissionais envolvidos no manejo de fauna silvestre. Consequentemente, a disseminação deste tipo de doenças possa ser mitigada entre a população.

\section{REFERÊNCIAS BIBLIOGRÁFICAS}

1. World Health Organization. Zoonoses. [Internet]: Genebra: World Health Organization; 2017 Feb [cited 2017 Feb 22]. Available from: http://www.who.int/topics/zoonoses/en/

2. Morse SS, Mazet JAK, Woolhouse P, Parrish CR, Carroll D, Karesh WB et al. Prediction and prevention of the next pandemic zoonosis. Lancet. 2012 Dec;380(9857):1956-65, doi: 10.1016/S01406736(12)61684-5. 
3. Karesh WB, Dobson A,Smith JO, Lubroth J, Dixon, MA, Bennett M et al. Ecology of zoonoses: natural and unnatural histories. Lancet. 2012 Dec;380(9857):1936-45, doi:10.1016/S0140-6736(12)61678-X.

4. Bidaisee S, Macpherson CNL. Zoonoses and one health: a review of the literature. J Parasitol Res. 2014 Jan; 874345, doi: 10.1155/2014/874345.

5. Jones KE, Patel NG, Levy MA, Storeugard A, Balk D, Gittleman JL et al. Global trends in emerging infectious diseases. Nature. 2008 Feb;451(7181):990-3, doi: 10.1038/nature06536.

6. Catão-Dias JL. Biossegurança na reintrodução de animais silvestres na natureza. Ci Vet Tróp. 2008 Jan;11:178-81.

7. Lemos ERS. Trabalho de campo com animais: procedimentos, riscos e biossegurança. Rio de Janeiro (Brasil): Fiocruz; 2014. Capítulo 5, Zoonoses como acidente de trabalho; p. 27-33.

8. Friend M. Disease emergence and resurgence: the wildlife-human connection. [Internet]. Virginia: Geological Survey; 2006 [cited 2016 Jan 20]. p. 7-16. Available from: https://www.nwhc.usgs.gov/publications/disease_emergence/Front.pdf

9. Wolfe ND, Dunavan CP, Diamond J. Origins of major human infectious diseases. Nature. 2007 May; 447(7142):279-83, doi: 10.1038/nature05775.

10. Foley JA, Defries R, Asner G, Barford C, Bonan G, Carpenter SR et al. Global Consequences of Land Use. Science, 2005 Jul;309(5734):570-4, doi: 10.1126/science.1111772.

11. Roche B, Guégan JF. Ecosystem dynamics, biological diversity and emerging infectious disease. C R Biol. 2011 May;334(5-6):385-92, doi: 10.1016/j.crvi.2011.02.008.

12. Lemos ERS, D'andrea PS. Trabalho de campo com animais: procedimentos, riscos e biossegurança. Rio de Janeiro: Fiocruz; 2014. 180 p.

13. Marvulo MFV, Carvalho VM. Tratado de animais selvagens: medicina veterinária. 2nd ed. São Paulo (Brasil): Roca; 2014. Capítulo 8, Zoonoses; p. 2194-206.

14. Krauss H, Weber A, Appel M, Enders B, Isenberg HD, Schiefer HG et al. Zoonoses: infectious diseases transmissible from animals to humans. 3nd ed Washington: ASM PRESS; 2003. 456 p.

15. Silva AS, Soares CDM, Gressler LT, Lara VM, Carregaro AB Monteiro SG. Criptosporidíase gastrintestinal em tamanduá-mirim (Tamandua tetradactyla). Rev Bras Zoo. 2009 Ago;10(2):175-77, doi: 10.34019/2596-3325.2008.v10.24066.

16. Miranda FR, Superina M, Vinci F, Hashimoto V, Freitas JC, Matushima ER. Serosurvey of Leptospira interrogans, Brucella abortus and Chlamydophila abortus infection in free-ranging giant anteaters (Myrmecophaga tridactyla) from Brazil. Pesq Vet Bras. 2015 Mai;35(5):462-5, doi: 10.1590/S0100736X2015000500013.

17. Nofs S, Abd-Eldaim, Thomas K, Toplon D, Rouse D, Kennedy M. Influenza virus A (H1N1) in giant anteaters (Myrmecophaga tridactyla). Emerg Infect Dis. 2009 Jul;15(7):1081-3, doi: 10.3201/eid1507.081574.

18. Frank R, Melaun C, Martins MM, Santos AL, Heukelbach J, Klimpel S. Tunga penetrans and further parasites in the giant anteater (Myrmecophaga tridactyla) from Minas Gerais, Brazil. Parasitol Res. 2012 Nov;111(5):1907-12, doi: 10.1007/s00436-012-3036-1.

19. Jorge S, Schuch RA, de Oliveira NR, da Cunha CEP, Gomes CK, Oliveira TL et al. Human and animal leptospirosis in Southern Brazil: A five-year retrospective study. Travel Med Infect Dis. 2017 Jul;18:4652, doi: 10.1016/j.tmaid.2017.07.010.

20. Machado FME, Coelho H E, Rezende RS. Plano de ação para o controle da leptospirose no zoológico municipal de Uberaba-MG. Biosci J. 2010 Nov;26(6):981-9.

21. Furtado MM. Estudo epidemiológico de patógenos circulantes nas populações de onça-pintada e animais domésticos em áreas preservadas de três biomas brasileiros: cerrado, pantanal e amazônia [tese]. São Paulo (SP): Universidade de São Paulo; 2010. 282 p, doi: 10.11606/T.10.2010.tde-05102012-134828.

22. Nogueira MF, Cruz TF. Doenças da capivara. Corumbá: Embrapa Pantanal; 2007. 74 p.

23. Barbosa AD, Silva NRM, Magalhães, DF. Zoonoses e saúde pública: riscos da proximidade humana com a fauna silvestre. Ci Vet Tróp. 2011 Jan;14(1):1-9.

24. Corpo de Bombeiros Militar de Minas Gerais. Instrução técnica operacional n ${ }^{\circ}$ 26/2016 Captura de animais. Belo Horizonte: Corpo de Bombeiros Militar de Minas Gerais; 2016. 34 p. Separata No 38 de 22 de setembro de 2016.

25. Bosch AS, Leong K, Musgrave K, Powers J, Wong D. Zoonotic disease risk perceptions and use of personal protective measures among wildlife biologists: An application of the health belief model. Hum Dimens Wildl. 2010 May;15:221-8, doi: 10.1080/10871200903460252.

26. Bosch AS, Musgrave K, Wong D. Zoonotic disease risk and prevention practices among biologists and other wildlife workers: results from a national survey, US National Park Service, 2009. J Wildl Dis. 2013 Jul;49(3):475-85, doi: 10.7589/2012-06-173.

27. Anderson ME. Contact precautions and hand hygiene in veterinary clinics. Vet Clin North Am Small Anim Pract. 2015 Mar;45(2):343-60, doi: 10.1016/j.cvsm.2014.11.003. 
28. Willians CJ, Scheffel JM, Elchos BL, Hopkins SG, Levine JF. Compendium of veterinary standard precautions for zoonotic disease prevention in veterinary personnel. National Association of State Public Health Veterinarians: Veterinary Infection Control Committee 2015. J Am Vet Med Assoc. 2015 Dez;247(11):1253-77, doi: 10.2460/javma.247.11.1252.

29. European Committee for Standardization - ECS. BS EN 388: protective gloves against mechanical risks. [Internet]. Hertfordshire: WISE Worksafe Ltd; 2017 Feb [cited 2017 Jul 29]. Available from: https://www.wiseworksafe.com/blog/view/understanding-en-388-2016-protective-gloves-against mechanical-risks

30. Comite Europeen Normalisation - CEN. SRPS EN 1082-3: protective clothing: gloves and arms guards protecting against cuts and stabs by hand knives: part 3: impact cut test for fabric, leather and other materials. [Internet]. 2010 May [cited 2017 Jul 29]. Available from: https://ec.europa.eu/growth/singlemarket/european-standards/harmonised-standards/personal-protective-equipment_en

31. Safety Tec. Consulta CA: portal de consulta de certificado de aprovação de equipamento de proteção individual (EPI). [Internet]. São Paulo: Safety Tec; 2017 Mai [citado 2017 Jul 29]. Disponível em: https://consultaca.com.

32. Nigam P, Srivastav A. Assessing occupational hazards among Indian wildlife health professionals. Veterinarski arhiv. 2011 Dec;81(6):731-41.

33. Baker WS, Gray GC. A review of published reports regarding zoonotic pathogen infection in veterinarians. J Am Vet Med Assoc. 2009 May;234(10):1271-8, doi: 10.2460/javma.234.10.1271.

34. Barros-Silva P, Fonseca L, Carneiro M, Vilges K, Oliveira S, Gonçalves R. Occupational risk of spotted fever: an evaluation of knowledge, attitudes and prevention practices among veterinary medicine students. Rev Patol Trop. 2014 Oct;43(4):389-97, doi.org/10.5216/rpt.v43i4.33597. 